Siriano, Discorsi di guerra. Testo, traduzione e commento di IMMACOLATA ERAMO, con una nota di LUCIANO CANFORA ( "Paradosis" 17), Edizioni Dedalo, Bari 2010, pp. 245, ISBN 978-88-220-5817-1.

Nel II libro del De oratore Cicerone lamentava una sorta di lacuna nella tradizione retorica antica per numerosi generi (in eodem silentio multa alia oratorum officia iacuerunt, II 15, 64); al primo posto dell'elenco erano le cohortationes, ossia gli incitamenti alla guerra che il generale-oratore aveva l'obbligo di porgere prima dell'inizio

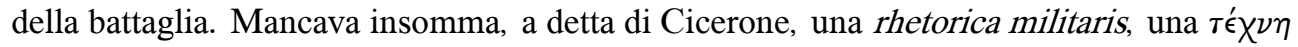
(more aristotelico, ça va sans dire) tutta declinata nell'ambito della tattica e della strategia di guerra. Il vuoto fu colmato in età bizantina dall'autore di un compendio piuttosto vasto, che collegava sostanzialmente tre trattati: una Rhetorica militaris, appunto, un De re strategica e uno scritto di tattica navale (testo che sopravvive acefalo, edito da Konrad Müller nel 1882 e poi più volte ripreso con il titolo di Navpaxíaı). L'editio princeps del primo dei tre (intitolato $\Delta \eta \mu \eta \gamma o p i ́ a \iota ~ \pi \rho о \tau \rho \epsilon \pi \tau$ каi) si deve a Hermann Köchly (18551856), che però non lo attribuisce ad alcuno scrittore, poiché la non estesa tradizione manoscritta è anonima. Una corposa serie di studi che attraversa la seconda metà dell'Ottocento - ed è poi ripresa anche nel XX secolo, fino all'attuale edizione a cura di Immacolata Eramo (=IE) - è quasi unanime nell'attribuire la redazione del compendio a

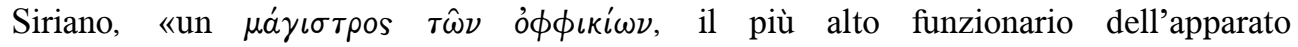
amministrativo bizantino, quasi certamente lo stesso Siriano la cui opera era consigliata da Costantino VII al figlio Romano quale utile viatico per le compagne militari $(\beta \iota \beta \lambda i a$

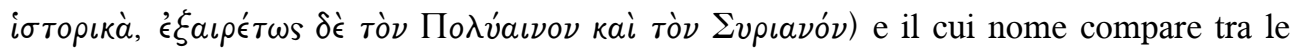
fonti dei Tactica di Niceforo Urano [...] e nella glossa a margine dei codici della recensio Laurentiana dei Tactica di Leone VI» (p. 15). La fonte manoscritta più importante dell'opera è infatti il Laurentianus LV.4 (L), codice membranaceo del X secolo che presenta una «raccolta degli scritti polemologici della Biblioteca imperiale di Costantinopoli, frutto di un'iniziativa ufficiale patrocinata da Costantino VII» (p. 24).

IE provvede a una nuova collazione dei testimoni, all'indagine dei loro rapporti interni, e quindi a nuova, equilibrata e documentata constitutio textus; in più fornisce la prima e unica traduzione italiana del manuale, con un commentario analitico ricchissimo di osservazioni e di collegamenti tra i Discorsi di guerra e tutta la letteratura retorica (e non soltanto) del mondo antico e bizantino. Il libro, insomma, è un'autentica edizione critica e al tempo stesso un aggiornato saggio di scienza retorica e polemologica. Sebbene non si conosca nulla della biografia dell'autore, il genere letterario - specifico e al tempo stesso sfaccettato - cui egli si dedica ha suscitato notevole interesse a partire dalla metà del XIX secolo. Come scrive la studiosa, «Maggiore fortuna ha sortito l'indagine sui 
caratteri letterari del compendio, in particolare $i$ suoi rapporti con il genere polemografico, per la sezione di tattica e poliorcetica, ma anche retorico, storico, stratagematico e biblico. Il risultato è un ricco repertorio di fonti, non sempre chiaramente esplicitate e identificabili, che abbraccia l'Iliade e il quarto libro dei Maccabei, e comprende anche Enea Tattico, Eliano, Filone di Bisanzio e Apollodoro di Damasco per l'ambito tattico e poliorcetico, Erodoto, Ctesia, Arriano e Polieno per aneddoti e riferimenti a episodi celebri del passato, il Vangelo di Giovanni e la pseudoepigrafa epistola paolina agli Ebrei per la professione di fede cristiana più volte manifestata nella Rhetorica militaris» (p. 15).

Dopo la Nota di Luciano Canfora (pp. 7-9) si dipana l'Introduzione (pp. 11-34), a sua volta articolata in due sezioni: nella prima IE traccia una sintetica storia del genere polemografico, giungendo fino a Siriano e alla sua epoca («pressappoco la dinastia dei Macedoni», p. 15); nella seconda dà conto dei testimoni manoscritti e dei loro rapporti, fino a ricostruire lo stemma codicum che li include tutti e che presuppone un archetipo. Direttamente da quest'ultimo derivano i due codici principali (e più antichi): il già citato Laurentianus (L) e l'Ambrosianus B 119 sup. (139) (A), anch'esso membranaceo del X secolo: il secondo «È l'unico manoscritto che riporta, benché notevolmente decurtate, tutte le sezioni del compendio di Siriano» (p. 28). Appoggiandosi soprattutto agli studi di Alphonse Dain, IE conclude che «L ed A provengano, indipendentemente l'uno dall'altro, da un comune antenato» (p. 29); gli altri testimoni sono apografi di questi due: copie, o copie di copie.

La sezione centrale del libro è occupata dal testo greco, con apparato critico di varianti e interventi correttivi, e dalla traduzione italiana (pp. 35-113). Il lettore, del resto, è già bene avvertito sui contenuti che si appresta a conoscere: «È una strategia che privilegia artificio, indugio, lentezza, dissimulazione, corruzione, in definitiva un approccio indiretto al conflitto, tale da ridurre al minimo tutti i rischi connessi. Più che lo scontro in armi, condotto in ossequio a norme predeterminate e a codici condivisi, sono importanti, anzi, determinanti, la valutazione delle forze, proprie e dell'avversario, le istanze motivazionali delle truppe, gli espedienti di natura psicologica, i rapporti tra belligeranti ed alleati, il principio del divide et impera, le trattative volte a scongiurare la battaglia, la razionalizzazione delle risorse umane e finanziarie» (p. 19). Nel porgere l'originale greco e le questioni relative alla tradizione manoscritta, IE interviene molto raramente con correzioni personali, preferendo stabilire la versione critica sulla base della tradizione e tutt'al più accettando alcuni dei numerosi aggiustamenti proposti da Köchly. Dal momento che le $\Delta \eta \mu \eta \gamma o p i a \iota$ di Siriano sono state oggetto di poca attenzione criticotestuale (nell'apparato l'unico nome di editore che compare è appunto quello di Köchly), ogni intervento di IE si rivela prezioso per la storia della ricezione, e merita di essere riportato nel dettaglio: in 7, 2 l'editrice propone un'espunzione nella frase oiov 


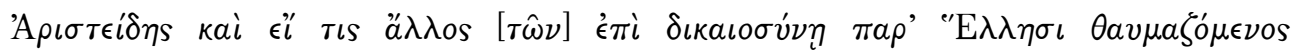
(all'interno del periodo: «Perciò anche molti per la loro giustizia sono beatificati ed onorati pure dopo la morte, come Aristide e quanti altri siano stati per la giustizia oggetto di ammirazione da parte dei Greci», pp. 44 s.), anche se in apparato utilizza una formula

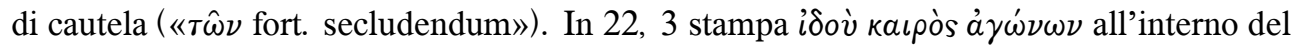
periodo che così traduce: «Se dunque le parole del generale sono vere, quanto cioè egli sosteneva a vostro vanto, ecco il momento della prova: ricordatevi dell'affetto che vi ho

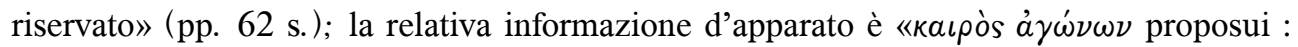

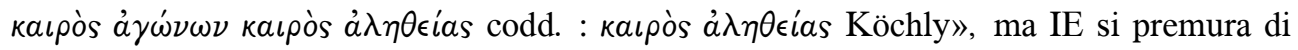
argomentare più diffusamente la propria scelta anche in nota: «Il testo dei manoscritti pare tradire un'origine glossematica; le due espressioni, kaıрòs á $\gamma \omega \dot{\nu} \omega \nu \nu$ da una parte e kaıpòs ả $\lambda \eta \theta$ єías dall'altra, sembrano essere alternative, o meglio l'una esplicativa

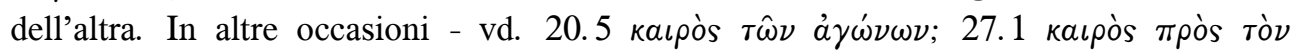

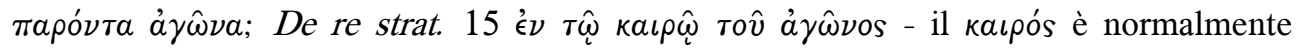
associato alla prova delle armi piuttosto che al 'momento della verità', pertanto sembrerebbe più fondato il sospetto che sia stata originariamente una glossa, entrata poi nel testo proprio alla luce della suggestione creata dalla veridicità o dalla verosimiglianza delle parole del generale citate poco prima» (p. 143). L'esempio dimostra come una correzione apparentemente non necessaria possa invece rivelarsi plausibile con la scorta di argomentazioni convincenti e con l'indicazione dell' usus scribendi dell'autore. Ancora più persuasiva la correzione di 37, 7 (6), che ha anche il pregio dell'economicità: IE

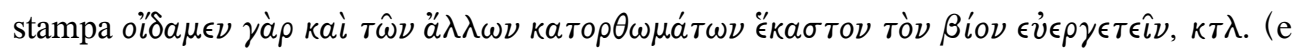
traduce: «Sappiamo, infatti, che anche ciascuno degli altri valori reca benefici alla vita, niente è tuttavia come la giustizia», pp. $82 \mathrm{~s}$.); in apparato si legge: «oi̊ $\delta a \mu \epsilon \nu$ supposui :

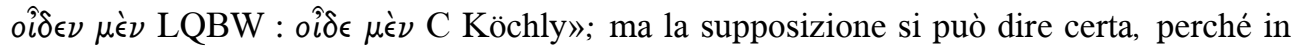
questo passaggio il pensiero dell'autore deve essere coniugato alla prima persona. L'ultimo intervento di IE sul testo di Siriano riguarda l'inscriptio del capitolo finale, insolitamente estesa e forse anche trasposta rispetto alla collocazione più adeguata:

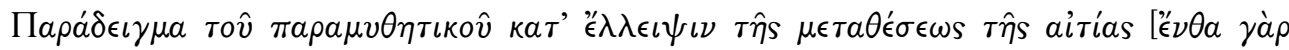

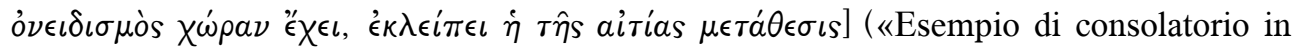
mancanza di spostamento della causa [quando infatti ha luogo un rimprovero manca lo spostamento della causa]», pp. 112 s.). La studiosa ipotizza che «il cap. 58 sia fuori posto (la sua più degna e naturale collocazione sarebbe all'interno o immediatamente dopo il cap. 56) e che il riferimento al rimprovero presente nella inscriptio tradisca un'origine glossematica: nato per superare l'impasse della ripresa a distanza causata dall'inidonea collocazione e per fornire un richiamo in sintesi e un collegamento anche con l'argomento subito precedente» (p. 194, che è l'ultima delle note).

П Н Г Н / F O N S I (2016), RESEÑAS, 170-174 
L'editrice ha denominato Note la sezione successiva, costituita appunto di 161 annotazioni esplicative numerate progressivamente (i numeri che vi rimandano sono dislocati nelle righe della traduzione italiana); è una scelta di grande umiltà, perché l'estensione complessiva (pp. 115-194, per di più in corpo minore) e l'accuratezza delle schede assurgono a esemplare commento di tutto il testo. Ed è un vero piacere, oltre che molto istruttivo, seguire di nota in nota IE nella perlustrazione del lessico tecnico della polemografia antica e dei suoi rapporti con gli autori di riferimento (da Tucidide ad Aristotele, da Demostene a Ermogene); attenendosi infatti all'ordine con cui sezioni e contenuti del discorso protrettico si presentano nel testo, IE ha modo di fornire la definizione e l'esemplificazione di argomenti generali e predisposizioni alla trattazione

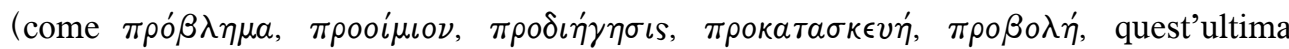
intesa come intentio criminis di un'azione giudiziaria), dello status quaestionis da cui si

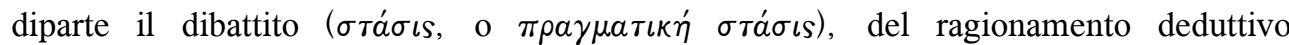

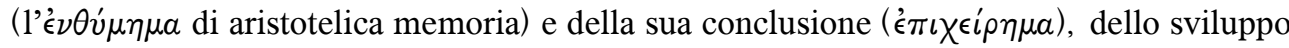

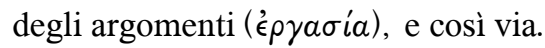

Costituisce davvero un unicum il compendio di Siriano, anche per la frequente congiunzione dell'esortazione guerresca con le leggi di vita prescritte dalla Bibbia e dalla cultura cristiana. Per questo motivo chi commenta il testo ha l'onere di spiegare $i$ numerosissimi exempla storici e letterari, che dai poemi omerici e dalla storia greca antica giungono fino alla storia romana. IE si disimpegna benissimo in questo compito, peraltro senza limitarsi alla nota puntuale e analitica; al contrario, il commento abbonda di chiarificazioni sintetiche, come quella inerente ai confini del genere praticato da Siriano: «La tattica si configura come una scienza a carattere eminentemente pratico, da sviluppare dopo aver bene appreso la capacità di maneggiare le armi (Pl. La. 182b), per questo non può che costituire solo una delle parti di cui si sostanzia l'arte del comando ed essere solo una porzione della $\sigma \tau \rho a \tau \eta \gamma_{i} a »($ p. 169). La ricchezza di terminologia tecnica è uno dei dati più interessanti del trattato; in effetti, gran parte degli accenni al lessico protrettico (tattico e strategico) sparsi nella letteratura retorica di più ampio raggio, in Siriano è spiegata dettagliatamente, soprattutto grazie ai numerosi esempi "da manuale" che l'autore ha raccolto. La guerra è dunque la realtà di fondo che motiva l'elaborazione retorica del buon generale al pari di tutti gli stratagemmi pratici che egli deve mettere in pratica sul campo di battaglia; ma è indispensabile quanto IE ricorda in proposito: «La civiltà bizantina, benché erede di una diuturna tradizione di guerre sul campo [...], ed essa stessa impegnata in uno stato di belligeranza con i confinanti pressoché ininterrotto, non giunse mai ad elaborare una giustificazione positiva della guerra (determinando in questo un solco ideologico rispetto all'Occidente, dove anche i teologi cristiani finirono per avallare la necessità di una guerra 'giusta' [...]), ma ricorse al tranquillizzante 


\section{Michele Curnis}

principio, già consacrato dalla tradizione [...], della guerra 'necessaria' in quanto difensiva» (p. 168).

Il volume si conclude con un Bibliografia (pp. 197-220) distinta in Edizioni e traduzioni del compendio di Siriano, Studi sul compendio di Siriano, Ulteriore bibliografia; seguono accuratissimi Indici (pp. 221-245), degli autori antichi, dei luoghi citati, dei nomi antichi, che includono anche tutti i riferimenti presenti nell'introduzione e nelle note della curatrice.

Un elogio finale deve essere dedicato anche all'eleganza editoriale del volume: in semplice brossura con sovraccoperta, ma realizzato con carta Lux cream 1, 8, gr. 70, con caratteri perfettamente leggibili e lineari sia nell'italiano sia nel greco, esso conferma l'impegno di rigore e di cura artigianale delle Edizioni Dedalo; con la sua tiratura limitata a 650 copie numerate a mano, anche questo è un libro di pregio del catalogo barese, oltre che un saggio di prima qualità storico-culturale e intellettuale. L'esemplare recensito è il n. 109 della produzione totale.

MiCHELE CURNIS 